Western University

Scholarship@Western

Department of Economics Research Reports

Economics Working Papers Archive

1983

\title{
On the Robustness of LM, LR and W Tests in Regression Models
}

Aman Ullah

Victoria Zinde-Walsh

Follow this and additional works at: https://ir.lib.uwo.ca/economicsresrpt

Part of the Economics Commons

Citation of this paper:

Ullah, Aman, Victoria Zinde-Walsh. "On the Robustness of LM, LR and W Tests in Regression Models." Department of Economics Research Reports, 8316. London, ON: Department of Economics, University of Western Ontario (1983). 
ISSN :

ISBN : $0-7714-0443-3$

RESEARCH REPORT 8316

ON THE ROBUSTNESS OF IM, LR AND $W$ TESTS IN REGRESSION MODELS

by

Aman U1lah and Victoria Zinde-Walsh

Ju1y, 1983

Department of Economics Librany

SEP 201983

University of Western Ontario 


\section{ON THE ROBUSTNESS OF LM, LR AND W}

TESTS IN REGRESSION MODELS

By Aman U1lah and Victoria Zinde-Walsh ${ }^{1}$

\section{INTRODUCTION}

In much applied as well as theoretical research work, the error terms in linear models are assumed to be normally and independently distributed. Such an assumption, it is well recognized, is often questionable and its violation may have varying effects in a variety of situations; see, for example, Gnanadesikan [6]. This has motivated us to analyze the robustness of Rao's score or Lagrangian multiplier (LM) test (see Rao [12], Aitchison and Silvey [1], and Silvey [14]), the likelihood ratio (LR) test and Wald's (W, [16]) test for testing restrictions of the univariate linear regression model with a joint multivariate student-t error distribution. ${ }^{2}$ The work on testing restrictions with LM, LR and $W$ in the usual normal regression model can be found in Savin [13], Breusch [3] and especially in a recent interesting paper by Evans and Savin [4]. Further, the robustness of the LR test has been attempted earlier by Zellner [18] and Ghosh and Sinha [5].

In this paper we have shown that, in the sma11 sample case, while the LR test is robust, the LM and $W$ tests are not robust when the errors are Student- $t$. For the large sample, however, all three tests are found to be robust. A variation of the $W$ test is also considered and it turns out to be non-robust. The implications of assuming the errors to be distributed as multivariate Student- $t$, rather than multivariate normal, are also discussed. It has been found that not all of Evans and Savin's [4] results carry through when the errors are Student- $t$. 


\section{THE MAIN RESULTS}

Let us consider the regression model

$$
y=x \beta+u
$$

where $y$ is an $n \times 1$ vector of observations on the dependent variable, $x$ is an nxp matrix of observations on the non-stochastic exogenous variables, $\beta$ is a pX1 coefficient vector and $u$ is an $n \times 1$ error vector.

It is known that if $\mathrm{u}$ is distributed as multivariate normal with mean vector zero and variance covariance matrix $\sigma^{2} I$, where $I$ is an $n \times n$ identity matrix, then the maximum likelihood (ML) estimator for $\beta$ is the ordinary least squares (OLS) estimator given by

$$
\text { (2.2) } \quad b=\left(X^{\prime} X\right)^{-1} x^{\prime} y
$$

Further, the ML estimator of $\sigma^{2}$ is given by

$$
s^{2}=\frac{1}{n}(y-x b)^{\prime}(y-x b)
$$

Also, for testing

$$
\begin{aligned}
& \mathrm{H}_{0}: \mathrm{R} \beta=\mathrm{r} \\
& \mathrm{H}_{1}: \mathrm{R} \beta \neq r,
\end{aligned}
$$

where $R$ is an mxp constant matrix of rank $m, r$ is an $m \times 1$ vector of constants and $R \beta=r$ is a set of $m$ linear restrictions on the coefficient vector $\beta$, the LR test criterion turns out to be

(2.5) LR $=-2 \log \ell$, with $\ell=\max _{R \beta=r, \sigma^{2}} L\left(\beta, \sigma^{2}\right) / \max _{\beta, \sigma^{2}} L\left(\beta, \sigma^{2}\right)=\left(s^{2} / \hat{\sigma}^{2}\right)^{n / 2}$

in which $\hat{\sigma}^{2}$ is the restricted ML estimator of $\sigma^{2}$

(2.6) $\quad \hat{\sigma}^{2}=\frac{1}{n}(y-x \hat{\beta})^{\prime}(y-x \hat{\beta})$ 
and $\hat{\beta}$ is the restricted $M L$ estimator of $\beta$ (see, for example, Vinod and Ullah [15, p. 62]) given by

(2.7) $\hat{\beta}=b-\left(X^{\prime} X\right)^{-1} R^{\prime}\left[R\left(X^{\prime} X\right)^{-1} R^{\prime}\right]^{-1}(R b-r)$.

Likewise the LM and $W$ test statistics are, respectively, given by (Evans and Savin [4, p. 739])

$$
L M=(R b-r)^{\prime}\left[R\left(X^{\prime} X\right)^{-1} R^{\prime}\right]^{-1}(R b-r) / \hat{\sigma}^{2}
$$

and

$$
W=(R b-r)^{\prime}\left[R\left(X^{\prime} X\right)^{-1} R^{\prime}\right]^{-1}(R b-r) / s^{2}
$$

We now consider the multivariate Student-t distribution of errors given as (see, for example, Zellner $[17,18]$ )

$$
\text { (2.10) } \quad f(u)=\frac{c}{\sigma^{n}}\left[1+\frac{\gamma^{-1}}{\sigma^{2}} u^{\prime} u\right] \quad,-\infty<u<\infty
$$

where

$$
\text { (2.11) } \quad c=\frac{\Gamma\{(n+\gamma) / 2\}}{(\pi \gamma)^{n / 2} \Gamma(\gamma / 2)}
$$

is a normalizing constant and $\gamma$ is the degrees of freedom parameter. ${ }^{3}$ The mean vector and the variance covariance matrix of this density are given as (2.12) $\quad E u=0$ and $E u u^{\prime}=\frac{\gamma \sigma^{2}}{\gamma-2} I, \quad \gamma>2 .^{4}$

Note that as $\gamma \rightarrow \infty$, the density in $(2.10)$ tends to multivariate normal with the mean vector zero and the variance covariance matrix $\sigma^{2} I$.

We introduce $L R_{t}, W_{t}$ and $L M_{t}$ to represent the $L R, W$ and $L M$ test statistics under the Student-t error given in (2.10). The main result of the paper can then be stated in the following Theorems. 
THEOREM 1: Under (2.10), the LR, LM and W test statistics for testing

$H_{0}: R \beta=r$ against $H_{1}: R \beta \neq r$ are given by

(2.13) $L R_{t}=L R, L M_{t}=\lambda^{-1} L M, W_{t}=\lambda W ; \quad 0<\lambda=\frac{n+\gamma}{n+\gamma+2}<1$

where $L R, L M$ and $W$ are as given in (2.5), (2.8) and (2.9), respectively.

PROOF: Using (2.10), write the 1ikelihood function for the parameters of the mode1 $y=X \beta+u$ as

$$
L\left(\beta, \sigma^{2}\right)=\frac{c}{\sigma^{n}}\left[1+\frac{\gamma^{-1}}{\sigma^{2}}(y-X \beta)^{\prime}(y-X \beta)\right]^{-\frac{1}{2}(n+\gamma)}=f(y)
$$

where $c$ is as given in (2.11). Then taking $\log$ on both sides of (2.14) we have

$$
\log L\left(\beta, \sigma^{2}\right)=\log c-\frac{n}{2} \log \sigma^{2}-\frac{(n+\gamma)}{2} \log \left[1+\frac{\gamma^{-1}}{\sigma^{2}}(y-X \beta)^{\prime}(y-X \beta)\right] .
$$

The first derivatives of $\log L\left(\beta, \sigma^{2}\right)$ with respect to $\beta$ and $\sigma^{2}$ are

$$
\begin{aligned}
& \frac{\partial}{\partial \beta} \log L\left(\beta, \sigma^{2}\right)=\frac{y^{-1}}{\sigma^{2}}(n+\gamma)\left[1+\frac{\gamma^{-1}}{\sigma^{2}}(y-X \beta)^{\prime}(y-X \beta)\right]^{-1} x^{\prime}(y-X \beta) \\
& \frac{\partial}{\partial \sigma^{2}} \log L\left(\beta, \sigma^{2}\right)=-\frac{n}{2 \sigma^{2}}+\frac{(n+\gamma) \gamma^{-1}}{2 \sigma^{4}}(y-\dot{x} \beta)^{\prime}(y-X \beta)\left[1+\frac{\gamma^{-1}}{\sigma^{2}}(y-X \beta)^{\prime}(y-X \beta)\right]^{-1} .
\end{aligned}
$$

Further, the following second derivatives of $\log L\left(\beta, \sigma^{2}\right)$ can easily be derived:

$$
\begin{aligned}
\frac{\partial^{2}}{\partial \beta \partial \beta^{\prime}} \log L\left(\beta, \sigma^{2}\right)= & \frac{\gamma^{-1}(n+\gamma)}{\sigma^{2}}\left(1+\frac{\gamma^{-1}}{\sigma^{2}}(y-X \beta)^{\prime}(y-X \beta)\right)^{-1}\left[-x^{\prime} X\right. \\
& \left.+\frac{2 \gamma^{-1}}{\sigma^{2}} x^{\prime}(y-X \beta)(y-X \beta)^{\prime} X\left(1+\frac{\gamma^{-1}}{\sigma^{2}}(y-X \beta)^{\prime}(y-X \beta)\right)^{-1}\right]
\end{aligned}
$$

$$
\begin{aligned}
\frac{\partial^{2}}{\partial \beta \partial \sigma^{2}} \log L\left(\beta, \sigma^{2}\right)= & \frac{(n+\gamma) \gamma^{-2}}{\sigma^{6}}\left[1+\frac{\gamma^{-1}}{\sigma^{2}}(y-X \beta)^{\prime}(y-X \beta)\right]^{-2}(y-X \beta)^{\prime}(y-X \beta) X^{\prime}(y-X \beta) \\
& -\frac{1}{\sigma^{4}} \gamma^{-1}(n+\gamma)\left[1+\frac{\gamma^{-1}}{\sigma^{2}}(y-X \beta)^{\prime}(y-X \beta)\right]^{-1} X^{\prime}(y-X \beta) .
\end{aligned}
$$

It is clear from (2.16) and (2.17) that the unrestricted ML estimators of $\beta$ and $\sigma^{2}$ are $b$ and $s^{2}$ as given in (2.2) and (2.3), respectively. Thus for $L\left(\beta, \sigma^{2}\right)$ in $(2.14)$ 


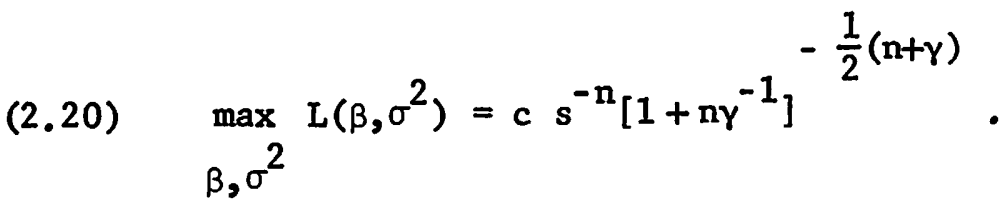

Similarly, it can be shown that

$$
\max _{R \beta=r, \sigma^{2}} L\left(\beta, \sigma^{2}\right)=c \hat{\sigma}^{-n}\left[1+n \gamma^{-1}\right]{ }^{-\frac{1}{2}(n+\gamma)}
$$

where $\hat{\sigma}$ is as given in (2.6). Using (2.20) and (2.21) we get the $L R_{t}$ which is the same as given in (2.5). This proves the first result in (2.13), and it compares with Zellner [18] and Ghosh and Sinha [5].

Now we derive the IM test statistic. First, notice that the right-hand side of (2.19) is an odd function of $u=y-X \beta$. Therefore

$$
E \frac{\partial^{2}}{\partial \beta \partial \sigma^{2}} \log L\left(\beta, \sigma^{2}\right)=0
$$

Further we observe that

$$
\begin{aligned}
E\left(1+\frac{\gamma^{-1}}{\sigma^{2}}(y-X \beta)^{\prime}(y-X \beta)\right)^{-1} & =\int_{y}\left(1+\frac{\gamma^{-1}}{\sigma^{2}}(y-X \beta)^{\prime}(y-X \beta)\right)^{-1} f(y) d y \\
& =\frac{c}{\sigma^{n}} \int_{u}\left(1+\frac{y^{-1}}{\sigma^{2}} u^{\prime} u\right) \\
& =\frac{\gamma}{(n+\gamma)}
\end{aligned}
$$

where the second equality has been obtained by using $u=y-X \beta$ and $f(y)=f(u)$, $-\infty<\mathrm{u}<\infty$; and the third equality has been obtained by noting that for $0<u<\infty$,

$$
\int_{u}\left(1+\frac{\gamma^{-1}}{\sigma^{2}} u^{\prime} u\right)^{-\frac{n+\gamma}{2}-1} d u=\left(\pi \gamma \sigma^{2}\right)^{n / 2} \frac{\Gamma\left(\frac{\gamma}{2}+1\right)}{\Gamma\left(\frac{n+\gamma}{2}+1\right)}
$$

from a result in Gradshteyn and Ryzhik [7, p. 623]. ${ }^{5}$ Also 
$(2.25)$

$$
\begin{aligned}
E(y-X \beta)(y-X \beta)^{\prime}\left(1+\frac{\gamma^{-1}}{\sigma^{2}}(y-X \beta)^{\prime}(y-X \beta)\right)^{-2} & =E_{u u^{\prime}}\left(1+\frac{\gamma^{-1}}{\sigma^{2}} u^{\prime} u\right)^{-2} \\
& =\frac{c}{\sigma^{n}} \int_{u} u u^{\prime}\left(1+\frac{\gamma^{-1}}{\sigma^{2}} u^{\prime} u\right)^{-\frac{n+\gamma}{2}-2} d u \\
& =\frac{\frac{\gamma^{2} \sigma^{2}}{(n+\gamma)(n+\gamma+2)} I,}{}
\end{aligned}
$$

where for $0<u<\infty$

(2.26) $\int_{u} u_{i}^{2}\left(1+\frac{\gamma^{-1}}{\sigma^{2}} u^{\prime} u\right)^{-\frac{n+\gamma}{2}-2} d u=\frac{1}{2} \pi^{n / 2}\left(\gamma \sigma^{2}\right)^{n / 2+1} \frac{\Gamma\left(\frac{\gamma}{2}+1\right)}{\Gamma\left(\frac{n+\gamma}{2}+2\right)}$

(see footnote 5 ).

Thus, from (2.18)

(2.27) $E\left[-\frac{\partial^{2}}{\partial \beta \partial \beta^{\prime}} \log L\left(\beta, \sigma^{2}\right)\right]=\frac{1}{\sigma^{2}}\left[X^{\prime} X-\frac{2}{n+\gamma+2} X \cdot X\right]=\frac{n+\gamma}{n+\gamma+2} \frac{1}{\sigma^{2}} X^{\prime} X=I(\beta)$

where $I(\beta)$ represents the information matrix of $\beta$. Further, since

$$
E \frac{\partial^{2}}{\partial \beta \partial \sigma^{2}} \log L\left(\beta, \sigma^{2}\right)=0
$$

from (2.22), the information matrix of $\beta$ and $\sigma^{2}, I\left(\beta, \sigma^{2}\right)$, will be a block diagonal matrix. Therefore, the LM test statistic can be written as

$$
\text { (2.28) } \quad L M_{t}=\hat{d}^{\prime} I^{-1}(\hat{\beta}) \hat{d}
$$

where $I(\hat{\beta})$ and $\hat{d}$ are $I(\beta)$ and $\frac{\partial}{\partial \beta} \log L\left(\beta, \sigma^{2}\right)$, respectively, evaluated at the restricted estimators $\beta=\hat{\beta}$ and $\sigma^{2}=\hat{\sigma}^{2}$. These are

(2.29) $\quad \hat{\mathrm{d}}=\frac{\mathrm{x}^{\prime} \hat{\mathrm{u}}}{\hat{\sigma}^{2}}$ 
(2.30) $I(\hat{\beta})=\frac{1}{\hat{\sigma}^{2}} \frac{(n+\gamma)}{(n+\gamma+2)} x^{\prime} x$,

where $\hat{\mathrm{u}}=\mathrm{y}-\mathrm{X} \hat{\beta}$ is the restricted residual and $\hat{\sigma}^{2}$ is as given in (2.6).

Substituting $(2.29)$ and $(2.30)$ in $(2.28)$ we get the LM test statistic for the multivariate-t case as

$$
\begin{aligned}
L M_{t}=\frac{n+\gamma+2}{n+\gamma} \hat{u}^{\prime} \frac{X\left(X^{\prime} X\right)^{-1} X^{\prime}}{\hat{\sigma}^{2}} \hat{u} & =\frac{n+\gamma+2}{n+\gamma} \frac{(R b-r)^{\prime}\left[R\left(X^{\prime} X\right)^{-1} R^{\prime}\right]^{-1}(R b-r)}{\hat{\sigma}^{2}} \\
& =\frac{n+\gamma+2}{n+\gamma} L M,
\end{aligned}
$$

where IM is the test statistic for the multivariate-normal case as given in (2.8). This gives the proof of the result for LM in Theorem 1 . Next, consider the $W$ statistic

(2.32) $\quad W_{t}=(R b-r)^{\prime}\left[R I^{-1}(b) R^{\prime}\right]^{-1}(R b-r)$,

where $I(b)$ is $I(\beta)$, given in (2.27), evaluated at the unrestricted estimators $\beta=b$ and $\sigma^{2}=s^{2}$. This is

(2.33) $\quad I(b)=\frac{1}{s^{2}} \frac{n+\gamma}{n+\gamma+2} X^{\prime} X$.

Substituting. $(2.33)$ in (2.32) then gives the result in (2.13) of Theorem 1.

Q.E.D.

THEOREM 2: For the $L R_{t}$, $L M_{t}$ and $w_{t}$ test statistics given in $(2.13)$ the following inequalities hold:

$(2.34)$

$$
\begin{array}{rlrr}
w_{t} & <L R_{t}<L M_{t} & \text { if } & \omega<\omega_{1}(\lambda) \\
L R_{t} & \leq w_{t}<L M_{t} & \text { if } & \omega_{1}(\lambda) \leq \omega<\omega_{2}(\lambda) \\
w_{t} \geq L M_{t}>L R_{t} & \text { if } & \omega_{2}(\lambda) \leq \omega<\omega_{3}(\lambda) \\
w_{t}>L R_{t} \geq L M_{t} & \text { if } & \omega & \geq \omega_{3}(\lambda),
\end{array}
$$

where $w=w / n, 0<\lambda=(n+\gamma) /(n+\gamma+2)<1$, and $\omega_{1}(\lambda), \omega_{2}(\lambda), \omega_{3}(\lambda)$ are, respectively, 
the unique positive roots (depending on $\lambda$ ) of equations

$$
\begin{aligned}
\lambda \omega-\log (1+\omega) & =0 \\
\lambda^{2} w-\omega(1+\omega)^{-1} & =0 \\
\lambda \log (1+\omega)-\omega(1+\omega)^{-1} & =0 .
\end{aligned}
$$

PROOF : We observe by using $(2.5),(2.8)$ and (2.9) in (2.13) that

$$
L R_{t}=n \log (1+\omega), L M_{t}=\lambda^{-1} n \omega(1+\omega)^{-1}, W_{t}=n \lambda \omega .
$$

Therefore, $W_{t}-L R_{t}=n(\lambda \omega-\log (1+\omega)), W_{t}-L M_{t}=n \lambda^{-1}\left(\lambda^{2} \omega-\omega(1+\omega)^{-1}\right)$ and $L R_{t}-\operatorname{LM}_{t}=n \lambda^{-1}\left(\lambda \log (1+\omega)-\omega(1+\omega)^{-1}\right)$. Notice that the equations (2.35) represent (up to a factor $n$ or $n \lambda^{-1}$ ) the differences $W_{t}-L R_{t}, W_{t}-L M_{t}$ and $L R_{t}-L M_{t}$, respectively.

Now write $w_{t}-\operatorname{LR}_{t}=n \Psi(\omega)$, where $\Psi(\omega)=\lambda \omega-\log (1+\omega)$. The function $\Psi(\omega)$ is convex, since $\Psi^{\prime \prime}(\omega)=(1+\omega)^{-2}>0$. Therefore $\Psi(\omega)=0$ (or the first equation in (2.35)) cannot have more than two roots. One root is $\omega=0$ and the other is a positive root, $\omega_{1}(\lambda)$. The existence of this unique positive root $\omega_{1}(\lambda)$ follows because for a small neighbourhood of zero where $\omega>0, \Psi(\omega) \simeq(\lambda-1) \omega<0$, and $\lim \Psi(\omega)=+\infty$. These features of $\Psi(\omega)$ also show that

$$
\begin{array}{lll}
w_{t}-L R_{t}<0 & \text { for } & \omega<\omega_{1}(\lambda) \\
w_{t}-L R_{t} \geq 0 & \text { for } & \omega \geq \omega_{1}(\lambda)
\end{array}
$$

Using similar steps, it can easily be verified that

$$
\begin{array}{lll}
w_{t}-L M_{t}<0 & \text { for } & \omega<\omega_{2}(\lambda) \\
w_{t}-L M_{t} \geq 0 & \text { for } & \omega \geq \omega_{2}(\lambda)
\end{array}
$$

and

$$
\begin{array}{lll}
L R_{t}-L M_{t}<0 & \text { for } & \omega<\omega_{3}(\lambda) \\
L R_{t}-L M_{t} \geq 0 & \text { for } & \omega \geq \omega_{3}(\lambda)
\end{array}
$$

where $\omega_{2}(\lambda)$ and $\omega_{3}(\lambda)$, respectively, are the unique positive roots of 
$W_{t}-L M_{t}=0$ and $L R_{t}-L M_{t}=0$ (or equivalently of the second and third equations in (2.35)). Notice that $\omega_{2}(\lambda)$ can be obtained explicitly.

Next, we estab1ish that

$$
\omega_{1}(\lambda)<\omega_{2}(\lambda)<\omega_{3}(\lambda)
$$

For this we first examine the inverse functions $\lambda_{1}(\omega)=\omega^{-1} \log (1+\omega)$, $\lambda_{2}(\omega)=(1+\omega)^{-1 / 2}, \lambda_{3}(\omega)=\omega[(1+\omega) \log (1+\omega)]^{-1}$ and show that

$\lambda_{1}(w)<\lambda_{2}(w)<\lambda_{3}(w)$. Let us consider the differences $\lambda_{3}(\omega)-\lambda_{2}(\omega)=\left(\omega-(1+\omega)^{1 / 2} \log (1+\omega)\right) /(1+\omega) \log (1+\omega)$ and $\lambda_{2}(\omega)-\lambda_{1}(\omega)=$ $\left(\omega-(1+\omega)^{1 / 2} \log (1+\omega)\right) / \omega(1+\omega)^{1 / 2}$. The signs of each of the differences coincide with the sign of the numerator $\left(w-(1+w)^{1 / 2} \log (1+w)\right)$, which is the same for the two differences. Notice that this numerator is convex, equals zero at $\omega=0$, and for sufficiently small $\omega>0$ it can be approximated by $w^{3} / 24>0$. Therefore it is positive, that is $\lambda_{2}(\omega)>\lambda_{1}(\omega)$ and $\lambda_{3}(\omega)>\lambda_{2}(\omega)$, for all $\omega>0$. Thus $\lambda_{1}(\omega)<\lambda_{2}(\omega)<\lambda_{3}(\omega)$ is true. In addition, by direct differentiation we can easily verify that each $\lambda_{i}(\omega)(i=1,2,3)$ is a monotonically decreasing function. This result along with $\lambda_{1}(\omega)<\lambda_{2}(\omega)<\lambda_{3}(\omega)$ establishes $(2.40)$ for $\omega_{i}(\lambda)$, $i=1,2,3$.

Fina $1.1 \dot{y}$, combining $(2.40)$ with the results in $(2.37),(2.38)$ and (2.39) the inequalities (2.34) of Theorem 2 follow.

Q.E.D.

It follows from our proof of Theorem 1 that $I^{-1}(\beta) \neq V(b)$ where $I(\beta)$ is as given in (2.27), and using (2.12), $V(b)=\sigma^{2} \gamma(\gamma-2)^{-1}\left(X^{\prime} X\right)^{-1} .^{6}$ Thus in the student-t case, a variation of $W_{t}$, given in (2.32), can be written as

$$
\text { (2.41) } \mathrm{W}_{1 t}=(\mathrm{Rb}-\mathrm{r})^{\prime}\left[\hat{\mathrm{RV}}(\mathrm{b}) \mathrm{R}^{\prime}\right]^{-1}(\mathrm{Rb}-\mathrm{r})=\lambda_{1} \mathrm{~W}_{2} \quad 0<\lambda_{1}=\frac{\gamma-2}{\gamma}<1 \text {, }
$$

where $\hat{V}(b)=s^{2} \gamma(\gamma-2)^{-1}\left(X^{\prime} X\right)^{-1}$ is the unrestricted estimator of $V(b)$, and $W$ is as given in (2.9). 
Likewise, we can obtain the asymptotic IM and W test statistics (Harvey

$[8, \mathrm{Ch} .5]$ as

(2.42) $\quad L M_{t}^{*}=\hat{d}^{\prime} I^{*-1}(\hat{\beta}) \hat{d}=L M$

and

(2.43) $\quad W_{t}^{*}=(R b-r)^{\prime}\left[R I^{*-1}(b) R^{\prime}\right]^{-1}(R b-r)=W$

where $I^{*}(\hat{\beta})=X^{\prime} X / \hat{\sigma}^{2}$ and $I^{*}(b)=X^{\prime} X / s^{2}$ are the restricted and unrestricted consistent estimators, respectively of $I^{*}(\beta)=n\left\{\lim _{n \rightarrow \infty} n^{-1} I(\beta)\right\}=\operatorname{n}\left\{\lim _{n \rightarrow \infty} n^{-1} \frac{X^{\prime} X}{\sigma^{2}}\right\}$, and $\hat{d}$ is as given in (2.29).

We observe that $W_{1 t}<W_{t}$ and $W_{t}^{*}>W_{t}$, where $W_{t}$ is as given in $(2.32)$. But when the distribution of errors is multivariate normal $(\gamma \rightarrow \infty), W_{1 t}=W=W_{t}^{*}$ because in this case $\hat{V}(b)=I^{-1}(b)=I^{*-1}(b)$.

\subsection{Remarks on the Results}

The following Remarks are based on the results in the Theorems. Remark 1 deals with the robustness of test statistics and Remarks 2,3 and 4 are about the comparison between our results and those of Evans and Savin [4] on inequality, power of exact test, and the conflict among the tests, respectively.

REMARK 1: It is clear from (2.13), (2.42) and (2.43), respectively, that $L R_{t}=L R, L M_{t}^{*}=L M$ and $W_{t}^{*}=W$. Hence the $L R$ and the asymptotic $L M$ and $W$ tests are robust against the multivariate-t distribution of errors. However, $L M_{t}=\lambda^{-1} L M \geq L M$ and $w_{t}=\lambda W \leq W, 0<\lambda=(n+\gamma) /(n+\gamma+2) \leq 1$, which implies that the IM and $W$ tests are not robust unless $n$ is large $(\lambda=-1){ }^{7,8}$ Nexto from (2.41) we observe that $W_{1 t}$, a variation of the $W$ test, is non-robust for both small and large samples. 
The above findings suggest that the LR, and the asymptotic LM and $W$ tests may be preferred to the $L M, W$ and $W_{1}$ tests with regard to the property of robustness.

REMARK 2: In the multivariate normal case Savin [13], Breusch [3], and Berndt and Savin [2] have shown the following inequality between the LR, LM and $W$ tests, (2.44) $\quad W \geq L R \geq L M$

Further, Evans and Savin [4] have indicated that when $|W / n|<1$

$$
W-L R \simeq L R-L M \simeq \frac{W^{2}}{2 n},
$$

that is, $W$ is greater than LR by approximately the same amount that LM is smaller than LR.

For the multivariate Student-t case, it can easily be verified, using (2.36), that when $|\omega|=|\mathrm{W} / \mathrm{n}|<1$

$$
\begin{aligned}
& W_{t}-L R_{t} \simeq \frac{W^{2}}{2 n}-W(1-\lambda) \\
& L R_{t}-L M_{t} \simeq \frac{W^{2}}{2 n} \frac{2-\lambda}{\lambda}-W \frac{1-\lambda}{\lambda}
\end{aligned}
$$

This shows that the Evans and Savin result in (2.45) does not go through in the multivariate-t case.

$$
\text { Next, noting that } \omega \geq \log (1+\omega) \text { and } \omega /(1+\omega) \leq \log (1+\omega) \text { we can }
$$

easily obtain from (2.36)

$$
\text { (2.47) } \quad \mathrm{W}_{t} \geq \lambda \mathrm{LR}_{\mathrm{t}} \geq \lambda^{2} \mathrm{LM}_{\mathrm{t}}
$$

This inequality is a weak analogue of the inequality (2.44) of Evans and Savin. It is, however, clear from Theorem 2 that (2.44) is not preserved under the multivariate-t distribution of errors. In fact, depending on the values of $\lambda$ and $\omega(\gamma, n$ and $w)$ we get rather different 
combinations of inequalities. ${ }^{9}$ Typically, for small values of $\omega$, the inequality (2.44) is reversed:

$$
\mathrm{w}_{\mathrm{t}}<\mathrm{LR} \mathrm{R}_{\mathrm{t}}<\mathrm{LM} \mathrm{M}_{\mathrm{t}}
$$

and for sufficiently large $\omega_{\text {, }}$ it holds in the same form as (2.44):

$$
W_{t} \geq L R_{t} \geq L_{t}
$$

Note, however, that when $n$ or $\gamma \rightarrow \infty(\lambda \rightarrow 1),(2.34)$ and (2.47) coincide with $(2.44)$.

Thus in general, the results in (2.44) and (2.45) for the multivariate normal case do not carry through for the LM and $W$ tests in the multivariate-t case. For the asymptotic LM and $W$ tests in (2.42) and (2.43), however, it is obvious that both (2.44) and (2.45) are valid.

REMARK 3: In the normal error case Evans and Savin [4, p. 740] have shown that all the LR, LM and $W$ tests are monotonic functions of the F-test statistic

$$
\text { (2.48) } \quad F=\frac{\text { RRSS - URSS }}{\text { URSS }} \frac{q}{m} \sim F(m, q) \text {, }
$$

where RRSS $=(y-X \hat{\beta})^{\prime}(y-X \hat{\beta})$, URSS $=(y-X b)^{\prime}(y-X b)$ and $q=n-p$. Therefore, the exact tests for each would have the same power function and there will be no conflict among them. These results would also hold for $L R_{t}, L_{t}$ and $W_{t}$ as each of these can be written as a function of $\mathrm{F}$ given below:

$$
\text { (2.49) } \quad \mathrm{W}_{t}=\lambda \frac{\mathrm{nm}}{\mathrm{q}} \mathrm{F}, \quad L M_{t}=\lambda^{-1} \frac{\mathrm{nmF}}{\mathrm{q}+\mathrm{mF}}, \quad L R_{t}=\mathrm{n} \log \left(1+\frac{\mathrm{m}}{\mathrm{q}} \mathrm{F}\right) .
$$

Notice that the right-hand side of (2.48) follows the central $F$ distribution under $H_{0}$ even when the errors follow the multivariate Student-t distribution (see Zellner [18, p. 401] and King [11]). Thus the test 
statistics in (2.49) can be used for testing the null hypothesis and for forming the confidence interval.

REMARK 4: The 1imiting distribution of each of the three statistics under $H_{0}$ is $\chi^{2}(m)$, a central chi-square with $m$ degrees of freedom. Let $z=\chi_{\alpha}^{2}(\mathrm{~m})$ be the critical value corresponding to the $\alpha \%$ critical region of $x_{m}^{2}$. Then, in the multivariate normal case, Evans and Savin [4] have shown that if this critical value is used the tests having the inequality relation $\mathrm{W} \geq$ LR $\geq$ LM will have the following characteristics. First, they will differ with respect to their sizes and powers in small samples, and there may be conflict between their conclusions. Second, when the sizes of the tests are corrected to be the same, the powers are approximately the same and the probability of conflict is nearly zero. The first characteristic of Evans and Savin's findings is in general true in the multivariate Student-t case. However, since the inequality relationship between $L R_{t}, L M_{t}$ and $W_{t}$ (see Theorem 2) is complicated, the relationship among the sizes of these tests and the possibilities of conflicts are quite different from those in Evans and Savin's case. For example, it follows from Theorem 2 that the size of the $\mathrm{w}_{t}$ test can be greater or less than that of the $\mathrm{LR}_{t}$ test depending on the values of $\omega$ and $\lambda$. In the Evans and Savin case, however, the size of the $W$ test is always greater than that of the LR test. We also note from (2.13) that, under $\mathrm{H}_{\mathrm{O}}$,

$$
P\left[L R_{t}>z\right]=P[L R>z], P\left[L M_{t}>z\right]>P[L M>z] \text { and } P\left[W_{t}>z\right]<P[W>z] \text {. }
$$

That is, the sizes of the $L R_{t}, L M_{t}$ and $w_{t}$ tests will be respectively, the same as, greater than, and less than, the corresponding LR, LM and W tests of the Evans and Savin case. These sizes can be calculated by 
following Evans and Savin [4, p. 741].

Since the distribution of the F statistic in (2.48) is unknown

under the alternative hypothesis, the second finding of Evans and Savin cannot be verified for $L R_{t}, L M_{t}$ and $W_{t}$ tests and it remains the subject of a future study. This observation is also true for the robust tests $\operatorname{LM}_{t}^{*}=L M$ and $W_{t}^{*}=W$ in $(2.42)$ and $(2.43)$.

University of Western Ontario 
REFERENCES

[1] Aitchison, J., and D. Silvey: "Maximum Likelihood Estimation of Parameters Subject to Restraints," Annals of Mathematical Statistics, 29 (1958), 813-828.

[2] Berndt, E., and N. E. Savin: "Conflict Among Criteria for Testing Hypotheses in the Multivariate Linear Regression Mode1," Econometrica, 45 (1977), 1263-1278

[3] Breusch, T. S.: "Conflict Among Criteria for Testing Hypotheses: Extension and Comment," Econometrica, 47 (1978), 203-208.

[4] Evans, G. B. A., and N. E. Savin: "Conflict Among the Criteria Revisited: The W, LR and IM Tests," Econometrica, 50 (1982), 737-748.

[5] Ghosh, M., and B. K. Sinha: "On the Robustness of Least Squares Procedures in Regression Models," Journal of Multivariate Analysis, $10(1980), 332-342$.

[6] Gnanadesikan, R.: Methods for Statistical Data Analysis of Multivariate Observations, John Wiley and Sons, New York, 7.977.

[7] Gradshteyn, I. S., and I. M. Ryzhik: Table of Integrals Series and Products, Academic Press, New York and London, 1980.

[8] Harvey, A. C.: The Econometric Analysis of Time Series, Philip Allan, Deddington, 1981.

[9] Kelker, D.: "Distribution Theory of Spherical Distributions and a Location Scale Parameter Generalization," Sankhya, A32 (1970), 419-430. 
[10] King, M. I.: "Some Aspects of Statistical Inference in the Linear Regression Mode1," Ph.D. Thesis, Department of Economics, University of Canterbury, Christchurch (1979).

[11] __ _ _ Robust Tests for Spherical Symmetry and Their Application to Least Squares Regression," Annals of Statistics, $\underline{8}$ (1980), $1265-1271$.

[12] Rao, C. R.: "Large Sample Tests of Statistical Hypotheses Concerning Several Parameters with Applications to Problems of Estimation," Proceedings of the Cambridge Philosophical Society, 44 (1947), $50-57$

[13] Savin, N. E.: "Conflict Among Testing Procedures in a Linear Regression Model with Autoregressive Disturbances," Econometrica, $44(1976), 1303-1315$.

[14] Silvey, S. D.: "The Lagrange MuItiplier Test," Annals of Mathematical Statistics, 30 (1959), 389-407. .

[15] Vinod, H. D., and A. U11ah: Recent Advances in Regression Methods, MarceI Dekker, New York, 1981.

[16] Wald, A.: "Tests of Hypotheses Concerning Several Paramters When the Number of Observations is Large," Transactions of the American Mathematical Society, 54 (1943), 426-482. .

[17] Zeliner, A.: An Introduction to Bayesian Inference in Econometrics, John Wiley and Sons, New York, 1971.

[18] Zellner, A.: "Bayesian and Non-Bayesian Analysis of the Regression Model with Multivariate Student-t Error Terms," Journal of the American Statistical Association, 71 (1976), 400-405. 


\section{Footnotes}

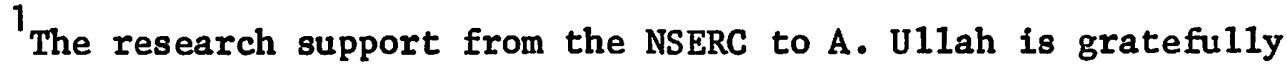
acknowledged. The authors are thankful to M. M. Ali, R. A. L. Carter and L. Magee for useful discussions. They also greatly benefitted from suggestions and comments by two anonymous referees which improved the exposition of the paper.

2 The multivariate Student-t distribution is chosen for our investigation since in this case the familiar $\mathrm{F}$ statistic has a central $\mathrm{F}$ distribution under the nu11 hypothesis. Also, in this case, explicit expressions of IR, IM and $W$ test statistics can be obtained which are useful in comparing with known results in the multivariate normal case.

3 Note that both the multivariate normal and the student-t are members of the class of elliptically symmetric distributions $f(u)=\sigma^{-n}|\Omega|^{-\frac{1}{2}} \phi\left(\frac{u^{\prime} \Omega^{-1} u}{\sigma^{2}}\right)$ with respect to the Lebesgue measure on $R^{n}$, where $u$ is an $n \times 1$ random vector, $\sigma^{2} \Omega$ is a positive definite matrix and $\phi()$ is a function on $[0, \infty)$. When $\Omega=I$ we get the class of spherically symmetric distributions. For further detail, see Kelker [9] and King [10, Ch. 3] among others.

${ }^{4}$ The results in this paper also hold for the more general case where the multivariate Student-t density is in the elliptical form $f(u)=c \sigma^{-n}|\Omega|^{-\frac{1}{2}}\left[1+\left(\gamma \sigma^{2}\right)^{-1} u^{\prime} \Omega^{-1} u\right]^{-(n+\gamma) / 2}$ for which Euu' $=\gamma(\gamma-2)^{-1} \sigma^{2} \Omega$. In this case, for any integral involving $u^{\prime} \Omega^{-1} u / \sigma^{2}$ we can use the non-singular transformation $v=P^{\prime} u$ such that $v^{\prime} v / \sigma^{2}=u^{\prime} \Omega^{-1} u / \sigma^{2}, d v=|\Omega|^{-\frac{1}{2}} d u$ and $E v v^{\prime}=\gamma(\gamma-2)^{-1} \sigma^{2} I$. 


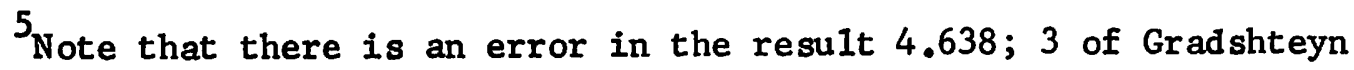
and Ryzhik [7]. The correct expression is

$$
\begin{aligned}
\int_{0}^{\infty} \int_{0}^{\infty} \ldots \int_{0}^{\infty} \frac{x_{1}^{p_{1}-1} \ldots x_{n}^{p_{n}{ }^{-1}}}{\left[1+\left(r_{1} x_{1}\right)^{q_{1}}+\ldots+\left(r_{n} x_{n}\right)^{\left.q_{n}\right]} d x_{1} \ldots d x_{n}\right.}= \\
\quad=\frac{\Gamma\left(p_{1} q_{1}^{-1}\right) \ldots \Gamma\left(p_{n} q_{n}^{-1}\right)}{q_{1} \ldots q_{n} r_{1} \ldots x_{n}} \frac{\Gamma\left(s-p_{1} q_{1}^{-1} \ldots-p_{n} q_{n}^{-1}\right)}{\Gamma(s)} .
\end{aligned}
$$

The integral in $(2.24)$ can alternatively be obtained by following ZeIIner $[17$, p. 384]

Whis shows that, under multivariate Student-t errors, the ML estimator $b$ does not achieve the Cramer-Rao Iower bound of the variance covariance since $V(b) \geq I^{-1}(\beta)$.

The evaluation of robustness for these tests appears complicated for the general case of elliptically symmetric distributions given in footnote 3. This is because in this case, the explicit forms of the information matrix required in both the LM and the $W$ tests are difficult to obtain. In a different context it follows from King's [11] work that the distribution of familiar $F$ test statistic for testing linear restrictions is robust against a class of elliptically symmetric distributions. However, this result does not imply the numerical robustness of the LR, LM and $W$ tests that we are considering in this paper.

${ }^{8}$ Notice that $\lambda \rightarrow 1$ even when $\gamma \rightarrow \infty$. In this case multivariate-t tends to multivariate normal and as expected $I M_{t} \rightarrow L M$ and $W_{t}\left(\right.$ also $\left.W_{1 t}\right) \rightarrow W$.

${ }^{9}$ Similar results can be developed for $w_{1 t}$ in (2.41). 